\title{
Study on the Application of P16/KI67 Protein in Screening of Cervical Cancer
}

\author{
Xuan $\mathrm{Li}^{1}$, Xiyong Ye ${ }^{1}$, Jianhua Gao ${ }^{1}$, Yingqun Liu ${ }^{2}$ \\ ${ }^{1}$ Jiangxi Medical College, Shangrao, Jiangxi, 334000 \\ ${ }^{2}$ Shangrao City People's Hospital, Shangrao, Jiangxi, 334000
}

Keywords: P16 and KI67, Screening of Cervical Cancer, Control Charts

\begin{abstract}
Cervical cancer is one of the most common malignant tumors of the gynecological department. In recent years, there has been a tendency to report the incidence of cervical cancer at home and abroad. The incidence of cervical cancer under 35 years of age is increasing, which seriously endangers women's health. Screening and prevention of cervical cancer is of great importance. The cervical intraepithelial neoplasia is a general term for a group of precancerous lesions closely related to cervical cancer, including cervical epithelial dysplasia and cervical carcinoma (CIS). It reflects the continuous development of cervical cancer development process, this period lasted for a long time, up to 10-20 years, and from atypical hyperplasia of cervix to carcinoma in situ and invasive cancer is a long-term development process, to prevent the occurrence of cervical cancer, The key is cervical screening of cervical intraepithelial neoplasia.
\end{abstract}

\section{Introduction}

Cervical cancer is one of the common malignancies in women, second only to breast cancer and colorectal cancer ranked third. China's new cases accounted for about $1 / 3$ of the world's incidence, ranking second in the world. In recent years, the Chinese government to encourage and strongly recommend cytology screening, making the incidence of cervical cancer has eased, but epidemiological shows that China's new cases of cervical cancer age was younger trend. Epidemiology has confirmed that HPV infection, especially high-risk HPV infection is a major risk factor for cervical cancer, high-risk HPV persistent infection is a necessary condition for cervical disease progression. In the prevention and treatment of cancer, cervical cancer is the only cause of the clear, can prevent malignant tumors. In the early lesions can be cured, but the symptoms are not obvious. With the development and popularization of cytology screening technology, more and more cervical precancerous lesions were found early and timely treatment. China's traditional screening of cervical cancer to take the method is "three steps" screening diagnostic procedures, that is, cytology a colposcopy a histological examination, but the three aspects of the diagnosis there is a certain Subjectivity. With the understanding of cervical lesions and HPV molecular biology research, in December 2014 cervical cancer screening program proposed a new three-step detection - cytology-colposcopy, high-risk type 16/18 HPV sieve The investigation will be the first threshold for cervical cancer screening, more and more immune indicators are better applied to the screening of cervical lesions. In 2012, the American College of Pathology (CAP) and the American Society of colposcopy and Cervical Pathology (ASCCP) guidelines indicate that p16INK4a antibody expression can reflect whether HPV infection affects cell cycle regulation. In the biological context of HPV, as a biomarker that suggests E6 / E7-driven cell proliferation, P16 is the only biomarker with sufficient data to support its use in the lower genital tract sample, the P16 gene is a cyclin-dependent kinase $\mathrm{CDK}$ ) to prevent the cells from G1 phase into the S phase, which play a role in inhibiting cell proliferation and mediating cell cycle. P16 overexpression has been shown to be strongly associated with the initiation of HP-HPV transformation infection. Pathological examination results as the final diagnosis and treatment of CIN lesions, but for the pathological diagnosis of CIN pathological diagnosis accuracy due to the existence of pathological factors subjective doctor, HE reproducibility of poor reproducibility, will lead to clinical treatment of CIN lesions. Statistics show that different pathologists in the normal tissue and CIN I diagnostic 
consistency is only $30 \%$ and $64 \%$, while the consistency of CIN II is less than $50 \%$. Therefore, the clinical needs more objective and more accurate criteria for judging. 2012 CAP / ASCCP has been P16 as a biological marker of cervical precancerous lesions, Klase and other studies have shown that, combined with P16 immunohistochemical staining to improve the diagnosis of cervical CIN lesions between the consistency and repeatability.

\section{Materials and Methods}

Collected from June to December 2014, outpatient TCT screening ASCS and above patients 671 cases, the routine HE staining pathological diagnosis: cervical cancer 375 cases, CIN269 cases, including 140 cases of CIN I, CIN II66 cases , 63 cases of CIN III, 27 cases of cervical cancer.

(TCT) was used to screen the cervical cytology.

The HPV subtype was detected simultaneously by the human papillomavirus (HPV) typing kit (PCR + membrane hybridization) provided by Hybrimax. $(16,18,58,31,33,35,39,45,51,56,59,68), 5$ low-risk types $(6,11,44,42,43)$ and 3 Asian common subtypes $(53,55$, CP8304) Experimental steps:

(1) secretions in the cell DNA separation and extraction

(2) polymerase chain reaction (PCR) amplification

(3) nucleic acid molecules rapid diversion hybridization

The immunohistochemical staining of this group of patients (Table 1-1 P16 + Ki-67 immunohistochemical score of pathological grading criteria) semi-quantitative score for the diagnosis of CIN pathological grading and HE medical records were compared. Cervical chronic inflammation is the diagnostic rate of $100 \%(375 / 375)$; CIN diagnostic rate of $95 \%(133 / 140)$. CIN II and CIN III overlap (34.88\%, 45/129), but HSI (129/129) can be diagnosed according to secondary classification. Statistical analysis with the exact probability method, $\mathrm{P}<0.001$, generally considered Kappa $\geqslant 0.75$ when the two consistency is better; $0.4 \leqslant$ Kappa $<0.75$ when the consistency of the general, Kappa $<0.4$ when the two consistency is poor. In this result, Kappa = 0.982 , indicating that the consistency in the actual sample is high.

In this group of patients with the level of increased HPV infection rate increased. The total infection rate of HPV was $73.62 \%(494 / 671)$. The infection rate was $62.4 \%(234 / 375)$ in the inflammatory group, $80 \%(112 / 140)$ in the CIN group and $95.45 \%$ in the CIN II group (63/66 ), The infection rate was $91.11 \%(41 / 45)$ in CIN II-III group, $94.44 \%(17 / 18)$ in CIN III group and 100\% $(27 / 27)$ in cancer group. In the HPV typing test, the first six subtypes were: type $1626.97 \%$ (181/671), 58 type 6.40\% (43/671), 18 type 4.02\% (27/671), type 52 3.43\% (23/671), type 2.98\% (4.27 / 671), type $2112.83 \%$ (19/671); mixed infection with high-risk mixed infection-based 110 cases, including high-risk mixed infection rate of 15.50\% 104/671). After the rank sum test, HPV16 infection and cervix CIN secondary classification of lesions was statistically significant $(\mathrm{P}=0.002)$.

Using the TBS diagnostic system of the Chinese Cancer Society in 2001, TBS reported normal or inflammatory cytology-negative; ASCUS, ASCUS-H and above were diagnosed as cytologically positive.

HE diagnostic criteria refer to the 2003 WHO female reproductive system of tumor pathology and genetics classification standards, by the three intermediate and above the name of the disease physician for all slices of independent reading film, the final use of the high rate of diagnosis with the results.

P16, Ki-67 immunohistochemical staining criteria to determine the standard: p16 expression in the nucleus and cytoplasm expression was positive; Ki-67 is mainly positive in the nucleus. P16INK4a, Ki-67 positive results were determined by staining results using semi-quantitative reports of intensity and distribution. Distribution of positive cells in the squamous epithelium vertical section, basal and basal cell staining for $1+, 1 / 3$ of the cells within the coloring of $2+$, more than $1 / 3$ less than $2 / 3$ coloring for $3+$, more than $2 / 3$ to full layer coloring for $4+$. Supplement: P16 is not expressed, only cytoplasmic staining or focal positive positive, Ki-67 no expression or only basal or basal side expression was negative (Ki-67 expression $1+$ as negative). According to Davied and other methods, by the pathologist with reference to P16, Ki-67 
immunohistochemistry combined score grading criteria for CIN to make immunohistochemical classification of the diagnosis.

\section{Combined Use of P16 and Ki-67 Semi-Quantitative Score in the Diagnosis of CIN Lesions}

In recent years, p16 and $\mathrm{Ki}-67$ in combination with cervical intraepithelial neoplasia classification diagnosis has become a hot topic, a large number of studies have shown that P16, Ki-67 combined use in the diagnosis of cervical CIN has a greater significance. Zhang Yan and other studies that p16, Ki- 67 combination can be used to identify and CIN lesions are easy to confuse the elderly atrophic changes, squamous metaplasia and as a CIN level of judgment, A kisser and Jaume think p16INK4a and Ki- 67 in the determination of cervical inflammatory response and LSIL diagnosis has a greater help. P16 as an important marker of cervical lesions, positive expression prompted HPV persistent infection and lead to abnormal cell proliferation. Ki-67 is a nuclear antigen of cell cycle progression and proliferative phase. It exists in all stages except the G0 phase in the cell cycle. Overexpression indicates that the cell is in the proliferative phase of the proliferative cycle. Usually the normal physiological function of cells P16 and Ki-67 will antagonize each other, will not appear at the same time. P16 alone used to predict high-grade lesions have a greater significance in the low-grade lesions and inflammatory lesions of the distinction and diagnosis of specificity is poor. The distribution of Ki- 67-positive cells is more important than their distribution. If $\mathrm{p}-\mathrm{Q}$ positive cells are located above the basal layer, even if the number of positive cells is small, the possibility of high-level CIN can not be excluded. In recent years, P16 and Ki-67 in the diagnosis of cervical lesions focused on the distinction between inflammatory and low-grade lesions or low-grade lesions and high-grade lesions. Jaume and so on will be applied to the cytology of colposcopic indications of the diversion, A Kisser and other studies used in the diagnosis of missing cases of inflammation screening, Li Siqi and so studied in the CIN I And CIN II in the identification, Calil and other studies of inflammatory reactive hyperplasia, atypical squamation is equal to the identification of CIN. In this study, the positive expression intensity of P16 and Ki-67 was quantitatively divided into negative, $1+, 2+, 3+, 4+$, and the expression of P16 and Ki-67 in different lesion levels was analyzed. Antibody expression intensity is between the scores of the combination of cervical lesions to make an objective pathological grading diagnosis. Comparison of the different grades combined with CIN pathological grading diagnosis and HE light microscope CIN pathological grading rate, we found that in the chronic cervicitis group, the two classification of all the diagnostic criteria. In the CIN I group, only 7 cases could not be diagnosed or diagnosed, the score was P16 negative, Ki-67 1+ 2 cases, P16 negative, Ki-67 3+ 2 cases, P16 1 +, Ki-67 3+ Example 1, P16 $2+$, Ki-67 3+, P16 3 +, Ki-67 1+. CIN II + can be diagnosed as HSIL by secondary classification. Statistical analysis with the exact probability method, $\mathrm{P}<0.001$, generally considered Kappa $\geqslant 0.75$ when the two consistency is better; $0.4 \leqslant$ Kappa $<0.75$ when the consistency of the general, Kappa $<0.4$ when the two consistency is poor. In this result, Kappa $=0.982$, indicating that the consistency in the actual sample is high. It suggests that the combined score can have a greater predictive significance of the lesion level, can be applied to CIN lesions screening or auxiliary diagnosis.

\section{Conclusion}

P16, Ki-67 immunohistochemical semi-quantitative score can objectively reflect the degree of CIN lesions. HE Scintigraphy and P16, Ki-67 immunohistochemical semi-quantitative score can improve the accuracy and reproducibility of CIN pathology, and provide a more reliable basis for clinical treatment. HPV infection in this group of patients with type differences, mainly to 16 $(26.97 \%), 58(6.40 \%)$ higher than $18(4.02 \%)$, should focus on prevention and treatment. P16 detection of cervix CIN lesions with HPV infection in the prognosis and prognosis has some tips. 


\section{Acknowledgements}

Title: TCT, HPV-DNA combined with P16, Ki67 detection in the diagnosis of cervical precancerous lesions and cervical cancer

Jiangxi Province Department of Education Science and Technology Research Project

Proj No.: GJJ151336

\section{References}

[1] Dong Airong. HPV induced cervical cancer mechanism research [J]. Chinese modern doctor, $2012(34)$

[2] Hao Haibo, XU Pei-quan.Study on the relationship between tumor suppressor gene p16 and malignancy [J]. Journal of Bengbu Medical College, 2012 (03)

[3] Yang Fan. HPV virus and cervical disease research progress [J]. Chinese community physician, $2011(28)$

[4] Jin Hua, Wang Fei. The role of HPV E7 gene in cell cycle [J]. Chinese Medical Journal, 2011 (01)

[5] Yang Shengli, Cao Fangqin. Significance of p16INK4A protein expression in cervical cancer [J]. Chinese Journal of Clinical Medicine, 2010 (31) 